\title{
Prevention of doxorubicin-induced
}

\section{cardiomyopathy using targeted MaFGF mediated by nanoparticles combined with ultrasound- targeted MB destruction}

\author{
This article was published in the following Dove Press journal: \\ International Journal of Nanomedicine \\ 26 September 2017 \\ Number of times this article has been viewed
}

\begin{abstract}
Xin-Qiao Tian ${ }^{1,2, *}$
Xian-Wei $\mathrm{Ni}^{3, *}$

$\mathrm{He}$-Lin $\mathrm{Xu}^{2, *}$

Lei Zheng ${ }^{4}$

De-Li ZhuGe 2

Bin Chen ${ }^{4}$

Cui-Tao Lu ${ }^{2}$

Jian-Jun Yuan ${ }^{1}$

Ying-Zheng Zhao

'Department of Ultrasonography, Henan Provincial People's Hospital (People's Hospital of Zhengzhou University), Zhengzhou, ${ }^{2}$ Department of Pharmacology, College of Pharmaceutical Sciences, Wenzhou Medical University, ${ }^{3}$ Department of Ultrasonography, The Second Affiliated Hospital of Wenzhou Medical University, ${ }^{4}$ Department of Ultrasonography, The First Affiliated Hospital of Wenzhou Medical University, Wenzhou, Zhejiang, China

*These authors contributed equally to this work
\end{abstract}

Correspondence: Ying-Zheng Zhao Chashan Gaojiao Yuanqu, College of Pharmaceutical Sciences, Wenzhou Medical University, Wenzhou, Zhejiang 325035, China

Email pharmtds@I63.com

Jian-Jun Yuan

Department of Ultrasonography, Henan Provincial People's Hospital (People's Hospital of Zhengzhou University), WeiWu Road No.7, Zhengzhou, Henan 450003, China Email yuan2373@163.com
Abstract: The present study seeks to observe the preventive effects of doxorubicin-induced cardiomyopathy (DOX-CM) in rats using targeted non-mitogenic acidic fibroblast growth factor (MaFGF) mediated by nanoparticles (NP) combined with ultrasound-targeted MB destruction (UTMD). DOX-CM rats were induced by intraperitoneally injected doxorubicin. Six weeks after intervention, the indices from the transthoracic echocardiography and velocity vector imaging showed that the left ventricular function in the MaFGF-loaded NP (MaFGF-NP) + UTMD group was significantly improved compared with the DOX-CM group. The increased malondialdehyde and decreased superoxide dismutase were observed in the DOX-CM group, while a significant increase in superoxide dismutase and a decrease in malondialdehyde were detected in the groups treated with MaFGF-NP + UTMD. From the Masson staining, the MaFGF-NP + UTMD group showed a significant difference from the DOX-CM group. The cardiac collagen volume fraction and the ratio of the perivascular collagen area to the luminal area number of terminal deoxynucleotidyl transferase-mediated deoxyuridine triphosphate nick end labelling positive cells in the MaFGF-NP + UTMD group decreased to 8.9\%, 0.55-fold, compared with the DOX-CM group (26.5\%, 1.7-fold). From terminal deoxynucleotidyl transferase-mediated deoxyuridine triphosphate nick end labelling staining, the results showed the strongest inhibition of apoptosis progress in MaFGF-NP + UTMD group. The immunohistochemical staining of the TGF- $\beta 1$ in MaFGF-NP + UTMD group reached 3.6\%, which was much lower than that of the DOX-CM group (12.6\%). These results confirmed that the abnormalities, including left ventricular dysfunction, myocardial fibrosis, cardiomyocytes apoptosis and oxidative stress, could be suppressed by twice weekly MaFGF treatments for 6 consecutive weeks (free MaFGF or MaFGF-NP+/UTMD), with the strongest improvements observed in the MaFGF-NP + UTMD group. Western blot analyses of the heart tissue further revealed the highest pAkt levels, highest anti-apoptosis protein (Bcl-2) levels and strongest reduction in proapoptosis protein (Bax) levels in the MaFGF-NP + UTMD group. This study confirmed the preventive effects of DOX-CM in the rats with MaFGF-NP and UTMD by retarding myocardial fibrosis, inhibiting oxidative stress, and decreasing cardiomyocyte apoptosis.

Keywords: doxorubicin-induced cardiomyopathy, MaFGF, UTMD, MaFGF-loaded

\section{Introduction}

Doxorubicin (DOX), an anthracycline drug, is one of the most effective and commonly used broad-spectrum anti-cancer agents against malignancies, such as breast and prostate cancers and childhood leukemia. ${ }^{1}$ Unfortunately, its clinical application is 
associated with dose-dependent and essential irreversible cardiotoxicity, which leads to the development of cardiomyopathy and congestive heart failure ${ }^{2}$ that is often lethal to the patients. The mechanisms of DOX-induced cardiotoxicity are multifactorial and involve oxidative stress with reactive oxygen species (ROS) generation and cell death through both apoptosis and necrosis. ${ }^{3}$

Acidic fibroblast growth factor (aFGF) is an important member of the FGF family, with a wide range of biological functions, including mitogenic and non-mitogenic activities, and it is expressed at high levels in the heart. ${ }^{4,5}$ However, aFGF has powerful mitogenic and proliferative influences and produces negative effects, such as tumor genesis and metastasis. ${ }^{6,7}$ Previous studies have shown that aFGF protects the cardiac function in myocardial diseases, such as diabetic cardiomyopathy and post-ischemic reperfusion, with its nonmitogenic activities. ${ }^{5,8}$ However, aFGF also has a potential tumorigenic ability given its mitogenic activities.

In recent years, a non-mitogenic aFGF-MaFGF was developed to weaken the mitogenic property while preserving the non-mitogenic activity, including antioxidation, antiapoptosis and cardioprotection. ${ }^{9}$ Therefore, MaFGF has a great potential in the high efficient treatment of myocardial injury induced by DOX. However, the reported administration of proteins and genes, including an intracoronary, intrapericardial or myocardial administration, are inconvenient and high risk in the clinical application. Highly efficient and safe delivery systems are needed for MaFGF in the treatment of DOX-induced cardiomyopathy (DOX-CM).

Recently, the technology of ultrasound-targeted MB destruction (UTMD) as a non-viral carrier system has become one of the most promising and safe strategies for the targeted delivery of proteins and genes. ${ }^{10-13}$ In addition, biodegradable nanoparticles (NP) can protect the proteins and genes from destruction by in vitro and in vivo factors. Therefore, highly efficient and safe delivery systems can be constructed by the combination of UTMD and biodegradable NP. In a previous study, we developed a novel material called "Poloxamer 188-grafted heparin copolymer," which has a high affinity for proteins and genes, especially for aFGF and MaFGF. ${ }^{9}$ An in vivo experiment showed that aFGF-loaded NP, in combination with UTMD in diabetic cardiomyopathy rat models effectively facilitates aFGF delivery into the myocardium and, therefore, improves cardiac function recovery. Therefore, the UTMD technique, combined with NP, may serve as a valuable approach to improve the cardiac specificity and efficacy of the MaFGF application for DOX-CM patients.
The present study aims to determine whether the combination therapy of the UTMD technique with novel MaFGF-loaded NP (MaFGF-NP) was effective in preventing DOX-CM in an animal model. The characterization of MaFGF-NP and microbubbles (MBs) was studied. To achieve an in-depth understanding of the therapeutic impact of the MaFGF-NP/UTMD technique, a broad range of commonly used pathophysiological indicators of the heart conditions was measured in a DOX-CM rat model. These measurements allowed for a thorough preclinical evaluation of the in vivo effects of 6 weeks of MaFGF-NP + UTMD treatment on the cardiac functions and related structural damages. Overall, this combination therapy provides DOX-CM patients an efficient adjuvant therapy to cure their pathologically damaged and dysfunctional hearts.

\section{Methods \\ Preparation and characterization of MaFGF-NP and MBs}

\section{Preparation of MaFGF-NP and MBs}

MaFGF (10 mg/mL) (Biological and Natural Medicine Development Center Limited Company of Wenzhou Medical University, China) was dissolved in $1 \mathrm{~mL}$ of $20 \% \mathrm{w} / \mathrm{v}$ Poloxamer 188-grafted heparin copolymer solution and was stirred and mixed. The resulting solution was added into $2 \mathrm{~mL}$ of $2.0 \% \mathrm{w} / \mathrm{v}$ gelatin solution to produce a homogeneous mixture. Under sonication $\left(110 \mathrm{w}, 15^{\circ} \mathrm{C}\right)$ using a probe sonicator for 15 second, D, L-glyceraldehyde was injected into the mixture until its final concentration reached $0.1 \% \mathrm{w} / \mathrm{v}$. The mixture solution was kept at $5^{\circ} \mathrm{C}$ and MaFGF-NP was formed by the cross-linking reaction under magnetic stirring at 2,500 rpm for $5 \mathrm{~h}$. Empty NP (blank NP, using Poloxamer 188-grafted heparin solution instead of MaFGF Poloxamer 188-grafted heparin solution in preparation) and free MaFGF solution (MaFGF dissolved in $0.9 \% \mathrm{NaCl}$ solution) were also prepared for comparison. Final MaFGF concentration in the MaFGFcontaining solutions (MaFGF-NP or MaFGF solution) was $6 \mu \mathrm{g} / \mathrm{mL}$.

An ultrasound contrast agent (SonoVue, Bracco Diagnostics, Princeton, NJ, USA) was used as MBs. Saline $5 \mathrm{~mL}$ was injected into the SonoVue bottle, which contained $59 \mathrm{mg}$ $\mathrm{SF}_{6}$ fluoride sulfur gases and $25 \mathrm{mg}$ lyophilized powder to form the MBs suspension, $90 \%$ of MB diameter $<6 \mu \mathrm{m}$, average diameter of $2.5 \mu \mathrm{m}$ and concentration was about $2-5 \times 10^{8}$ bubbles $/ \mathrm{mL}$. Oscillation shaking of the MBs was performed before mixing the MaFGF or MaFGF-NP, and shaking was continued in the process of treatment. 


\section{Characterization of MaFGF-NP and MBs}

The morphology of MaFGF-NP was assessed under transmission electron microscopy (TEM) at $120 \mathrm{kV}$. Briefly, $10 \mu \mathrm{L}$ of the NP solution, in water, was dropped on to a carbon-coated grid. It was dried in the ventilated place for $2 \mathrm{~h}$ and negatively stained with $2 \%$ phosphotungstic acid with further drying for 2 days. For the MB characterization, $100 \mathrm{mg}$ of ultrasound contrast agent powder was added into $1 \mathrm{~mL}$ of phosphate buffered saline (PBS) $(0.01 \mathrm{M}, \mathrm{pH}=7.4)$ and was gently shaken for $3 \mathrm{~min}$. After the powder totally dispensed, $10 \mu \mathrm{L}$ of the suspension was added dropwise to the slide, and the morphology was observed under the optical microscope. Nano measurer software (Wenzhou, China) was used for determining the size of the MBs.

\section{Animal studies \\ DOX-CM animal model}

This study was approved by the Animal Care and Use Committee of Wenzhou Medical University. All animalhandling procedures were performed according to the Guide for the Care and Use of Laboratory Animals from the National Institutes of Health. Seventy male Sprague Dawley rats (42-50 days, $180-220 \mathrm{~g}$, average $200 \pm 15 \mathrm{~g}$ ) were prefed for 1 week to adapt to the environment, and then, they were randomized into 7 groups ( $\mathrm{n}=10$ per group). The rats in group 2-7 were intraperitoneally injected with DOX (SigmaAldrich Co., St Louis, MO, USA) at $3.0 \mathrm{mg} / \mathrm{kg}$ on Mondays for 6 weeks to induce DOX-CM. The rats of group 1 were injected with saline instead. All the animals were maintained in stainless steel cages under $12 \mathrm{~h}$ cycles of light and dark, and the room temperature was kept at $20^{\circ} \mathrm{C}-25^{\circ} \mathrm{C}$ with a relative humidity of $50 \%$.

\section{Groups and treatments}

The treatments were processed after the injection of DOX on every Thursday and Saturday for 6 consecutive weeks. With the following treatment, the cardioprotective effect of MaFGF-NP combined with the UTMD technique was investigated. For all the animals, the MaFGF/MaFGF-NP and MBs were administered via a caudal vein injection, and the treatment prescription is shown in Table 1 and as follows: Group 1 (blank control): $1 \mathrm{~mL}$ of saline solution without MBs and ultrasound (US); Group 2 (DOX-CM group): $1 \mathrm{~mL}$ of saline solution without MBs and US; Group 3 (MaFGF group): $\operatorname{MaFGF}(3 \mu \mathrm{g} / \mathrm{kg})$ in $1 \mathrm{~mL}$ of saline solution without MBs and US; Group 4 (MaFGF-NP group): MaFGF-NP $(3 \mu \mathrm{g} / \mathrm{kg}$ ) in $1 \mathrm{~mL}$ of saline solution without MBs and US; Group 5 (UTMD group): $1 \mathrm{~mL}$ of the MB suspension
Table I Treatment prescription of animal

\begin{tabular}{lllll}
\hline & Doxorubicin & MaFGF & MaFGF-NP & UTMD \\
\hline Group I & $(-)$ & $(-)$ & $(-)$ & $(-)$ \\
Group 2 & $(+)$ & $(-)$ & $(-)$ & $(-)$ \\
Group 3 & $(+)$ & $(+)$ & $(-)$ & $(-)$ \\
Group 4 & $(+)$ & $(-)$ & $(+)$ & $(-)$ \\
Group 5 & $(+)$ & $(-)$ & $(-)$ & $(+)$ \\
Group 6 & $(+)$ & $(+)$ & $(-)$ & $(+)$ \\
Group 7 & $(+)$ & $(-)$ & $(+)$ & $(+)$ \\
\hline
\end{tabular}

combined only with US; Group 6 (MaFGF + UTMD): a mixture of free MaFGF ( $3 \mu \mathrm{g} / \mathrm{kg}$ ) and $1 \mathrm{~mL}$ of MB suspension combined with US; and Group 7 (MaFGF-NP + UTMD group): a mixture of MaFGF-NP $(3 \mu \mathrm{g} / \mathrm{kg})$ and $1 \mathrm{~mL}$ of MB suspension combined with US.

\section{Ultrasound-targeted MB destruction}

Each rat was anesthetized with an intraperitoneal injection of $350 \mathrm{mg} / \mathrm{kg}$ chloral hydrate, allowing for spontaneous respiration. The thoracic region was shaved, and the rats were placed in the left recumbent position. The 512C Sequoia color Doppler diagnosis system with RT-MCE contrast mode (Siemens Medical Solutions, Mountain View, CA, USA) was utilized to generate the UTMD effect. The linear array transducer (15L8-w probe, $12-14 \mathrm{MHz}$ ) was placed over the heart of the rat $($ depth $=3.0-4.0 \mathrm{~cm})$, and the probe and skin were filled with the coupling agent. Then, the parasternal short-axis view at papillary muscle level was obtained, and the ultrasonic apparatus was converted into RT-MCE contrast mode. A 20-gauge cannula was inserted into the caudal vein, and the experimental solution was infused. There was no MB signal before the filling of the MB and MaFGF-containing treatment. When a large number of MBs were seen filling the heart, the MB destruction function key (the key for controlling the bursting of MBs) attached to the machine was employed to disrupt the MBs for UTMD (US exposure duration per time $=10 \mathrm{~s}$, repeat three times with off intervals of $1 \mathrm{~s}$ to allow refill of the tissue with more MBs). After the bursting, the MBs completely disappeared in the heart.

\section{Transthoracic echocardiography and velocity vector imaging}

All the subjects underwent an echocardiographic study (Siemens Sequoia 512) in the first week before the injection of DOX (ie, before treatment) and the seventh week (ie, the week after treatment). The rats were anesthetized with an intraperitoneal injection of $350 \mathrm{mg} / \mathrm{kg}$ chloral hydrate sodium pentobarbital and were placed in the 
left recumbent position, and the needle electrodes were attached to the limbs for the electrocardiogram recording. The linear array transducer (15L8-w probe, 12-14 MHz) was placed over the heart of the rat $($ depth $=3.0-4.0 \mathrm{~cm})$, filling the coupling agent between the probe and skin, and the conventional echocardiography of every rat was taken. From the short-axis view at the middle level of left ventricle (LV), verified by the presence of prominent papillary muscles, the left ventricular internal dimension-diastole (LVIDd), left ventricular internal dimension-systole (LVIDs), left ventricular ejection fraction (LVEF) and left ventricular fractional shortening (LVFS) were captured (all the parameters were averaged of 3 consecutive cycles), as well as the QRS wave and T wave as a sign of systole and diastole.

After the conventional echocardiography, velocity vector imaging was applied, and the spatial resolution was reduced appropriately to improve the frame frequency $(60-90 \mathrm{~Hz})$. The continuous 3 cardiac beats of the 2-dimensional images were acquired using the Sequoia 512. All the images were stored as standard Digital Imaging and Communication in Medicine format images on magneto-optical disc (MO) and were analyzed offline in Siemens Sygno US Workplace 3.01 (Sygno VVI, Siemens Medical Solutions). In this study, the LV wall at mid-level (papillary muscle level), from the short-axis view, was divided into 6 segments according to the standard 16-segment model of the American Society of Echocardiography. The segments of the LV wall were plotted, the endocardial and epicardial borders were manually identified in a single frame of a cine-loop, and the borders in other frames were automatically generated, allowing the operators to alter any of those contours. Next, the segmental mean peak systolic radial velocity (Vs), the peak radial strain (RS) and the peak radial strain rate (RSr) were obtained from the velocity, the strain, and the strain rate curves provided by SygnoVVI.

\section{Histological and molecular analyses}

After all the experiments were completed, the body weight (BW) of rats was measured, and then, the rats were sacrificed. The heart tissues were rapidly excised and weighed, and the heart weight (HW) and the ratio of the HW/BW were measured. One part of left ventricular tissue samples from the papillary muscle level was obtained and stored in $2.5 \%$ glutaraldehyde for electron microscope studies. Another part of samples was fixed in 10\% formalin for paraffin sectioning. The remaining tissues were cryopreserved in liquid nitrogen until subsequent analysis, such as Western blotting and other molecular analyses.
Measurement of cardiac malondialdehyde (MDA) and superoxide dismutase (SOD)

The MDA level in the heart tissue was estimated by thiobarbituric acid (TBA) assay kit (Nanjing Jiancheng Bioengineering Institute, Nanjing, China). Briefly, the homogenate of the myocardium was centrifuged at $3,000 \mathrm{rpm}$ at $4^{\circ} \mathrm{C}$ for $15 \mathrm{~min}$ to collect the supernatant. The clear supernatant solution was mixed with the prepared TBA. The color that developed was measured at $532 \mathrm{~nm}$ against a blank with a spectrophotometer (BioPhotometer plus, Eppendorf AG, Hamburger, Germany). The values are expressed as nanomoles per mg protein.

The activity of SOD in the heart was examined according to xanthine oxidase method provided by the standard assay kit (Nanjing Jiancheng Bioengineering Institutea). The red substances caused by the xanthine-xanthine oxidase system were detected by the absorbance at $550 \mathrm{~nm}$. The values are expressed as units per mg of protein.

\section{Hematoxylin and eosin staining}

To visualize the cardiomyocyte architecture, the cardiac tissue was sectioned into $4 \mu \mathrm{m}$ thick slices for H\&E staining to evaluate the general morphology of the myocardium. The sections were observed, and the images were captured using a Nikon ECLPSE 80i (Nikon Corporation, Tokyo, Japan).

\section{Masson staining}

Cardiac collagen was detected using the Masson trichrome stain. Briefly, paraffin-embedded cardiac tissue sections were processed at $4 \mu \mathrm{m}$ for dewaxing and rehydration and were stained with standard Masson trichrome to examine myocardial interstitial fibrosis and perivascular fibrosis. With this staining, the normal myofiber was stained red, while the collagen was stained blue. Digital pictures were taken with identical exposure settings for all the sections. The cardiac collagen volume fraction $(\mathrm{CVF})$ and the ratio of the perivascular collagen area to the luminal area (PVCA/LA) were obtained by use of computer-assisted image analysis software (Image-Pro plus 6.0 imaging software, Media Cybernetics, Silver Spring, MD, USA). CVF was obtained by calculating the mean ratio of connective tissue to the total tissue area of the perivascular collagen. The collagen surrounding an intramyocardial coronary artery was considered to be perivascular collagen. PVCA/LA was obtained by examining the mean ratio of perivascular collagen area to coronary artery luminal area of cut cross-sectional vessel specimens.

\section{Transmission electron microscopy}

A portion of the left ventricle was cut into $1 \mathrm{~mm}$ fragments and was fixed in $2.5 \%$ glutaraldehyde over night for electron 
microscopic examination. The myocardial tissues were fixed in $1 \%$ osmic acid and were dehydrated in a series of acetone washes, and the tissues were embedded in Araldite for coronal sections. The myocardial tissue was sectioned at $1 \mu \mathrm{m}$ and was stained with toluidine blue. Ultra-thin sections were cut in from this block and were observed under a JEM-1230 (JEOL, Tokyo, Japan) transmission electron microscope.

\section{Immunohistochemical staining}

Four micrometer thick paraffin sections from the middle ring of the $\mathrm{LV}$ were stained with a polyclonal rabbit anti-transforming growth factor (TGF)- $\beta 1$ antibody $(1: 1,000$, Abcam, Cambridge, UK). The staining was visualized by a reaction with 3,3-diaminobenzidine (1:20) (DAB; SigmaAldrich Co). The sections were then lightly counterstained with Mayer's hematoxylin, dehydrated, and xylene-based mounted under glass cover slips. The negative controls were treated as aforementioned except that the primary antibody was replaced with PBS. The brown colored sites were quantified at a final magnification of $200 \times$ with an optical microscope connected to a video camera. TGF- $\beta 1$ staining intensity was analyzed using Image-pro Plus 6.0 software. Twenty-five random fields per section were analyzed and combined to obtain a final value for each section.

Terminal deoxynucleotidyl transferase-mediated deoxyuridine triphosphate nick end labelling (TUNEL) staining

The $4 \mu \mathrm{m}$ thick sections of cardiac tissue were stained for TUNEL to identify DNA fragmentation. All the sections were deparaffinized in xylene, rehydrated by ethanol washes, permeabilized with $0.1 \%$ PBS-Triton X-100 for $30 \mathrm{~min}$, and washed 3 times for $10 \mathrm{~min}$ at room temperature. Then, the apoptotic cells were detected by the In Situ Cell Death Detection Kit (Roche Molecular Biochemicals, Mannheim, Germany) according to the manufacturer's instructions. The images were visualized using a fluorescence microscope (Olympus, Tokyo, Japan). To quantitatively examine the numbers of apoptotic cells, the TUNEL-positive cells were counted. The average number of TUNEL-positive cells per section was calculated from the values obtained by counting 30-40 random sections throughout the lesion site of each animal, with 3 animals examined per group.

\section{Western blotting assay}

The cardiac tissues were lysed using radioimmunoprecipitation assay buffer (Beyotime Institute of Biotechnology, Shanghai, China) containing a protease inhibitor cocktail (Sigma, St louis, MO, USA). The proteins were isolated from the homogenized tissues with the TRIzol reagent (Invitrogen, Carlsbad, CA, USA) using standard Invitrogen protocols. The protein concentration was measured by the Bradford protein assay. Equal amounts of protein $(20 \mu \mathrm{g})$ were electrophoresed through a $10 \%$ sodium dodecyl sulfate polyacrylamide gel electrophoresis and were transferred to polyvinylidene difluoride membranes (Millipore Company, Billerica, MA, USA). The membrane was blocked with 5\% skim milk powder in PBS overnight. Then, the membrane was incubated with primary antibodies, including protein kinase B (Akt, 1:1,000 dilution), Ser473-phospho-Akt (pAkt 1:1,000 dilution), B-cell lymphoma 2 (Bcl-2, 1:1,000 dilution), and Bcl-2-associated $\mathrm{X}$ protein (Bax, 1:1,000 dilution) primary antibodies (Cell Signaling Technology, Boston, MA, USA). The glyceraldehyde 3-phosphate dehydrogenase antibody (GAPDH, 1:1,000 dilution; Kangchen, Shanghai, China) was used as a loading control. The proteins were then treated with goat antirabbit IgG-horseradish peroxidase as the secondary antibody $(1: 5,000)$ (Santa Cruz Biotechnology, Santa Cruz, CA, USA) and were visualized with using the ChemiDic ${ }^{\mathrm{TM}} \mathrm{XRS}+$ Imaging System (Bio-Rad Laboratories Inc., Hercules, CA, USA). The signals were quantified using Quantity One software.

\section{Statistical analysis}

The data are expressed as the mean \pm SE. Statistical significance was examined using a one-way analysis of variance followed by Tukey-multiple comparison test. All the statistical analyses were performed using SPSS version 19.0 (SPSS Inc., Chicago, IL, USA). The differences were considered statistically significant when the $P$-value was $\leq 0.05$.

\section{Result Characterization of the MaFGF-NP and MBs}

The TEM image of the MaFGF-NP (Figure 1A) indicated that the MaFGF-NP were spheres with a mean size of $73.5 \mathrm{~nm}$. In addition, the size distribution of the MaFGF-NP was measured by Dynamic Light Scattering Detector (DLS; Waters Q2000, New Castle, DE, USA). with an average hydrodynamic particle diameter of $91.3 \mathrm{~nm}$ (Figure 1B). In the light microscope, the MBs were round shaped. The particle size and distribution was measured and analyzed, and the average particle size was $4.66 \mu \mathrm{m}$.

\section{Improvement of the HW/BW ratio}

The BW, HW and the HW/BW ratio of the animals were measured to evaluate the general effects of MaFGF-NP/ UTMD therapy on DOX-CM. As expected, all 6 groups injected with DOX had lower BWs compared with the 

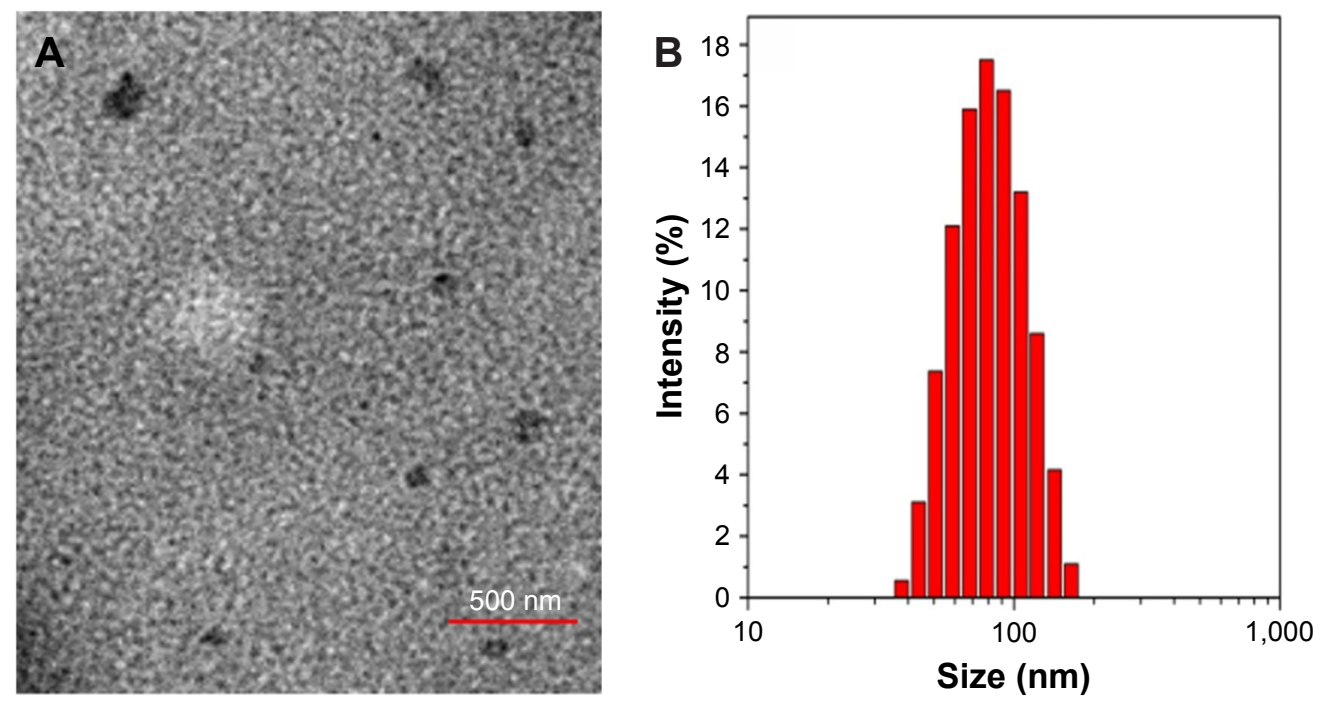

Figure I Characterization of the MaFGF-NP and the MBs.

Notes: (A) TEM of the MaFGF-NP. (B) Size distribution of the MaFGF-NP.

Abbreviations: MaFGF-NP, mitogenic acidic fibroblast growth factor-nanoparticles; TEM, transmission electron microscopy.

control regardless of the treatments $(P>0.05)$. However, Figure 2 shows that the HW of the DOX-CM group and the UTMD group increased compared with the other 4 groups with MaFGF or MaFGF-NP, but this was not significant $(P>0.05)$. In contrast, the groups treated with
MaFGF-NP, MaFGF + UTMD and MaFGF-NP + UTMD possessed lower HW/BW than the untreated DOX-CM animals $(P<0.05)$ and UTMD only. The results indicated that the MaFGF treatment with NP or/and UTMD modified cardiomyopathy.
A

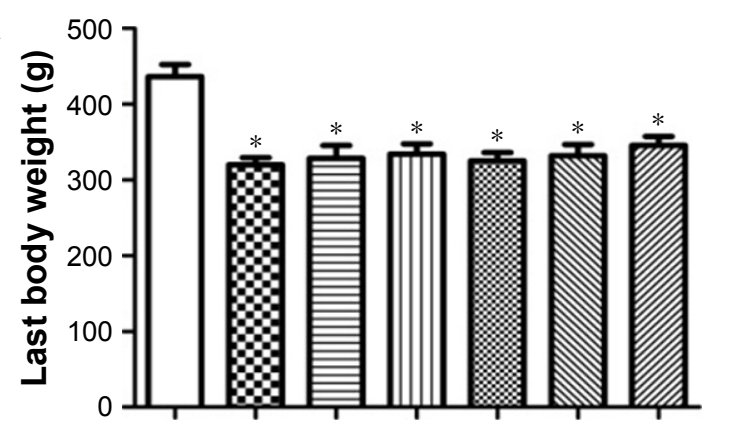

B

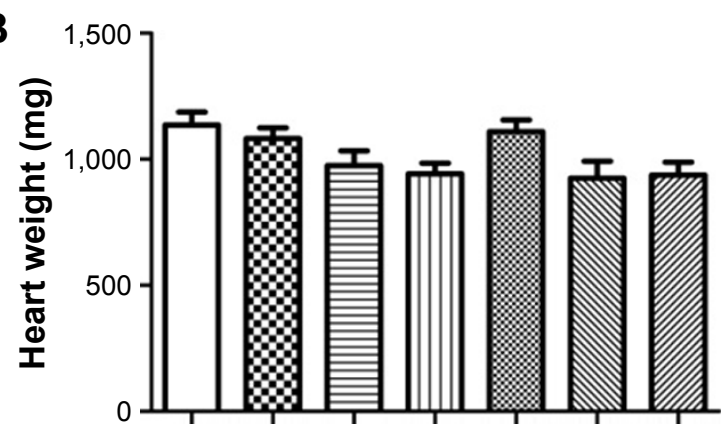

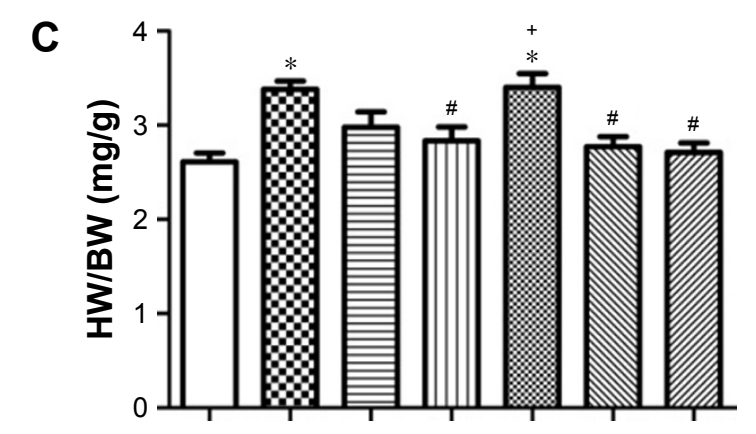

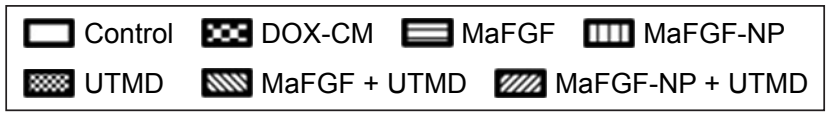

Figure 2 Quantitative analysis of the last body weight of the rats $(\mathbf{A})$, the heart weight of the rats $(\mathbf{B})$ and the heart-to-body weight ratio of the rats $(\mathbf{C})$. Notes: ${ }^{*} P<0.05$ vs control group; ${ }^{*} P<0.05$ vs DOX-CM group; ${ }^{+} P<0.05$ vs MaFGF-NP + UTMD group.

Abbreviations: BW, body weight; DOX-CM, doxorubicin-induced cardiomyopathy; HW, heart weight; MaFGF-NP, mitogenic acidic fibroblast growth factor-nanoparticles; UTMD, ultrasound-targeted MB destruction. 


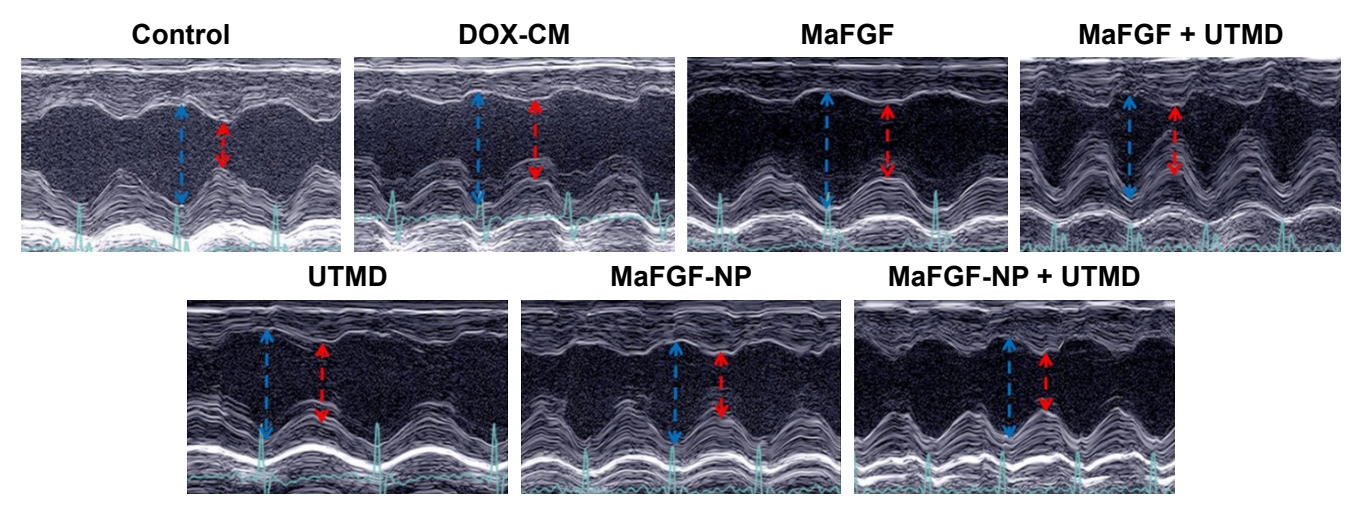

Figure 3 Echocardiograms of the left ventricle in $M$ mode.

Notes: The left ventricular internal dimension-diastole is depicted by a blue arrow, and left ventricular internal dimension-systole is depicted by a red arrow.

Abbreviations: DOX-CM, doxorubicin-induced cardiomyopathy; MaFGF-NP, mitogenic acidic fibroblast growth factor-nanoparticles; UTMD, ultrasound-targeted MB destruction.

\section{Prevention of left ventricle dysfunction in DOX-CM rats}

Two-dimensional parameters, including LVIDd, LVIDs, LVEF and LVFS were measured by transthoracic echocardiography to monitor the improvement of global myocardial contractile function (Figure 3). As shown in Table 2, the LVIDd and LVIDs of the DOX-CM group that were injected with DOX for 6 weeks and did not receive any treatment, showed great increase compared with the control group $(P<0.05)$, while LVEF and LVFS were significantly decreased $(P<0.05)$. The results illustrated that the cardiotoxicity of DOX seriously impaired the rat's global myocardial contractile function. However, a significant increase in the LVEF and LVFS $(P<0.05)$, as well as the decrease in the LVIDd and LVIDs $(P<0.05)$, was detected in the groups after the MaFGF treatment. Moreover, the group receiving MaFGF-NP combined with the UTMD treatment showed the highest LVEF and LVFS, as well as lowest LVIDd and LVIDs, among all the groups treated with MaFGF, which indicated that the combined treatment with MaFGF-NP and UTMD had the best effect on the prevention of cardiac dysfunctions in the DOX-CM rats by reversing all the indices close to a normal level.
The data of myocardial velocity, strain and strain rate measured by velocity vector imaging (VVI) are shown in Table 3. After 6 weeks, the values of Vs, RS and RSr were noticeably decreased $(P<0.05)$ to various extents in the DOX-CM animals, indicating deterioration of ventricular function injured by DOX. In comparison, after treatment with a combination of MaFGF-NP and UTMD, minimal differences compared with the control group were observed, and all 3 parameters were significantly larger than the other groups including the remaining 3 MaFGF-treated groups. Taken together, MaFGF-NP + UTMD led to the least ventricular dysfunction in the DOX-CM animals.

\section{Attenuation of DOX-induced oxidative stress}

As shown in Figure 4, the myocardial levels of MDA in the DOX-CM group were greatly increased compared with the control group $(P<0.05)$, while the activity of SOD significantly decreased $(P<0.05)$. However, the significant increase in SOD $(P<0.05)$ and the decrease in MDA $(P<0.05)$ were detected in the groups treated with MaFGF-NP,

Table 2 Results of transthoracic echocardiography

\begin{tabular}{|c|c|c|c|c|}
\hline Groups & LVIDs (mm) & LVIDd (mm) & LVEF (\%) & LVFS (\%) \\
\hline Control & $3.72 \pm 0.48$ & $7.19 \pm 0.56$ & $84.32 \pm 3.49$ & $48.4 I \pm 4.05$ \\
\hline DOX-CM & $5.54 \pm 0.45^{*,+}$ & $7.70 \pm 0.3 I^{*}$ & $59.69 \pm 2.50 *$ & $27.80 \pm 2.5 I^{*}$ \\
\hline MaFGF & $4.74 \pm 0.33^{*, \#,+}$ & $7.05 \pm 0.32^{\#}$ & $67.28 \pm 4.48^{*, \#,+}$ & $32.84 \pm 3.58^{*, \#,+}$ \\
\hline MaFGF-NP & $4.50 \pm 0.44^{* \#,++}$ & $6.98 \pm 0.35^{\#}$ & $69.65 \pm 3.91 \%, \#,+$ & $35.19 \pm 4.01 *, \#,+$ \\
\hline UTMD & $5.5 \mathrm{I} \pm 0.57^{*,+}$ & $7.69 \pm 0.37^{*,+}$ & $58.87 \pm 4.56^{*,+}$ & $27.38 \pm\left. 2.9\right|^{*,+}$ \\
\hline MaFGF + UTMD & $4.30 \pm 0.35^{*, \#,+}$ & $7.08 \pm 0.34^{\#}$ & $73.04 \pm 3.15^{*, \#,+}$ & $37.52 \pm 4.67^{* \#,++}$ \\
\hline MaFGF-NP + UTMD & $3.96 \pm 0.46^{\#}$ & $6.84 \pm 0.40^{\#}$ & $78.96 \pm 2.73 * \#$ & $42.04 \pm 3.67^{*, \#}$ \\
\hline
\end{tabular}

Notes: $* P<0.05$ vs control group; ${ }^{*}<0.05$ vs DOX-CM group; $+P<0.05$ vs MaFGF-NP + UTMD group. Data presented as mean \pm SD.

Abbreviations: d, diastole; DOX-CM, doxorubicin-induced cardiomyopathy; LVFS, left ventricular fractional shortening; LVID, left ventricular internal dimension; LVEF, left ventricular ejection fraction; MaFGF-NP, mitogenic acidic fibroblast growth factor-nanoparticles; s, systole; UTMD, ultrasound-targeted MB destruction. 
Table 3 Results of velocity vector imaging

\begin{tabular}{llll}
\hline Groups & Vs (cm/s) & RS (\%) & RSr ( I/s) \\
\hline Control & $1.08 \pm 0.35$ & $28.27 \pm 6.29$ & $3.36 \pm 1.20$ \\
DOX-CM & $0.45 \pm 0.13^{*}$ & $7.06 \pm 3.17^{*,+}$ & $1.46 \pm 0.76^{*}$ \\
MaFGF & $0.69 \pm 0.21^{*}$ & $10.57 \pm 3.0 I^{*, \#,+}$ & $1.77 \pm 0.67^{*,+}$ \\
MaFGF-NP & $0.78 \pm 0.26^{*, \#}$ & $16.02 \pm 4.45^{*, \#,+}$ & $2.47 \pm 0.65^{\#}$ \\
UTMD & $0.46 \pm 0.16^{*}$ & $7.30 \pm 2.90^{*,+}$ & $1.54 \pm 0.62^{*,+}$ \\
MaFGF + UTMD & $0.82 \pm 0.32^{\#}$ & $16.73 \pm 5.17^{*, \#,+}$ & $2.70 \pm 0.75^{\#}$ \\
MaFGF-NP + UTMD & $0.88 \pm 0.34^{\#}$ & $23.56 \pm 5.07^{*,+}$ & $2.96 \pm 1.15^{\#}$ \\
\hline
\end{tabular}

Notes: $* P<0.05$ vs control group; ${ }^{*}<<0.05$ vs $D O X-C M$ group; $+P<0.05$ vs MaFGF-NP + UTMD group. Data is presented as mean \pm SD.

Abbreviations: DOX-CM, doxorubicin-induced cardiomyopathy; MaFGF-NP, mitogenic acidic fibroblast growth factor-nanoparticles; RS, radial strain; RSr, radial strain rate; UTMD, ultrasound-targeted MB destruction; Vs, systolic radial velocity.

MaFGF + UTMD and MaFGF-NP + UTMD. Moreover, the group that received MaFGF-NP combined with the UTMD treatment showed the lowest MDA, as well as the highest SOD, among all the 3 groups, which indicated that the MaFGF-NP + UTMD had the best antioxidation effect in the DOX-CM rats.

\section{Attenuation of the impairment of myocardium induced by DOX}

The H\&E staining (Figure 5) of the DOX-CM group showed disorganization of the muscle fibers, marked interstitial edema and focal cytoplasmic vacuolation compared with the control group. All 4 groups with MaFGF or MaFGF-NP showed different moderate degrees of edema, disorganization and vacuolation compared with the DOX-CM rats. However, the lowest degree was observed in the MaFGF-NP + UTMD group compared with other treatment.
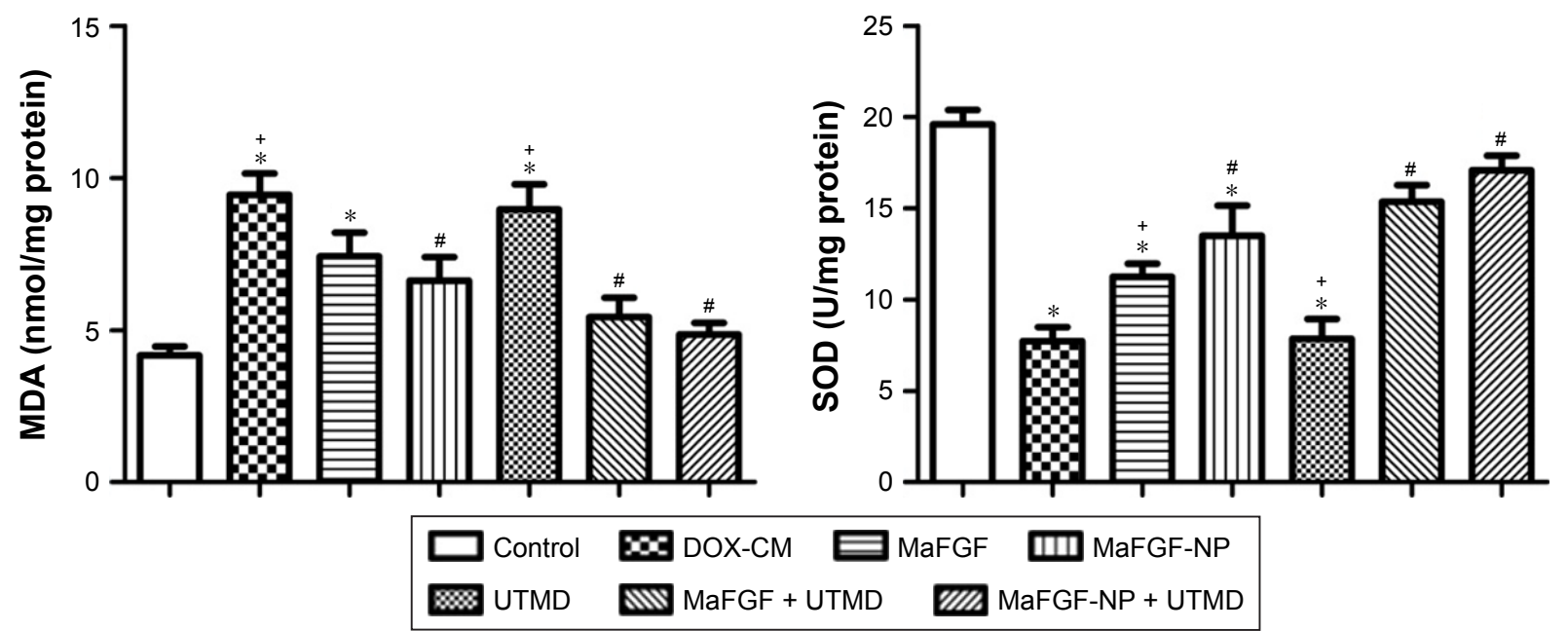

Figure 4 Quantitative analysis of the myocardial level of MDA (left) and SOD (right).

Notes: ${ }^{*} P<0.05$ vs control group; ${ }^{*} P<0.05$ vs DOX-CM group; ${ }^{+} P<0.05$ vs MaFGF-NP + UTMD group.

Abbreviations: DOX-CM, doxorubicin-induced cardiomyopathy; GAPDH, glyceraldehyde 3-phosphate dehydrogenase; MDA, malondialdehyde; MaFGF-NP, mitogenic acidic fibroblast growth factor-nanoparticles; SOD, superoxide dismutase; UTMD, ultrasound-targeted MB destruction.

\section{Attenuation of DOX-induced cardiomyocyte interstitial fibrosis and perivascular fibrosis}

Red or deep red normal muscle fibers and blue myocardial interstitial collagen fibers were observed through a microscopic examination of Masson trichrome stained tissues shown in Figure 6. Cardiac remodeling, especially an increase in cardiac fibrosis, was observed in the hearts of the mice administered DOX only compared with the controls and other animals $(P<0.05)$. The increased CVF $(26.5 \%)$ and PVCA/LA (1.66-fold) demonstrated the altered and disorganized collagen network structure in the interstitial and perivascular areas. In comparison with the DOX-CM group, CVF and PVCA/LA were significantly reduced in the groups treated with MaFGF $(18.62 \%$, 1.19-fold) or MaFGF-NP (14.36\%, 0.89-fold). Among the MaFGF treatment groups, there was a lower interstitial and perivascular fibrosis in the MaFGF-NP + UTMD group (8.92\%, 0.55-fold) compared with the other MaFGF groups without NP or UTMD. It should be noted that UTMD alone did not reduce the severity and extent of DOX-induced fibrosis.

\section{Effect of MaFGF-NP/UTMD on the expression of TGF- $\beta$ I}

As shown in Figure 7, the TGF- $\beta 1$ expression in the myocardial interstitium of the DOX-CM group was significantly increased compared with the myocardial interstitium of the control rats $(P<0.05)$. Immunohistochemistry revealed that the MaFGF/MaFGF-NP + UTMD treated groups exhibited 


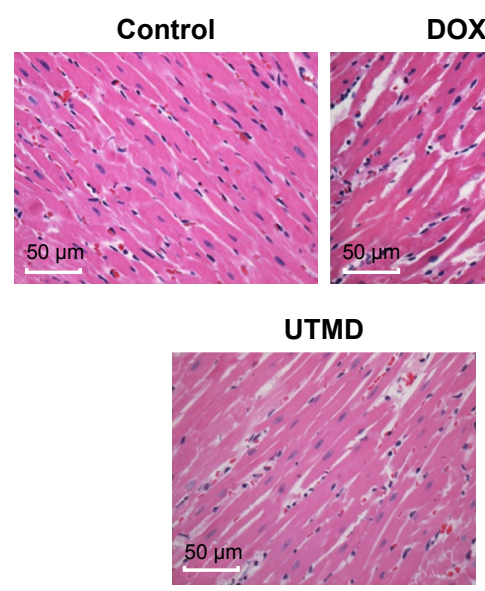

DOX-CM

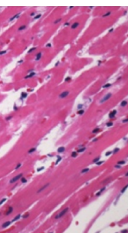

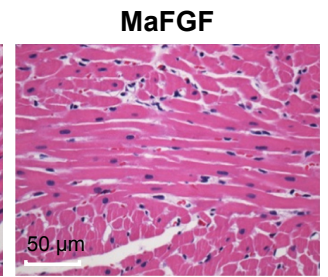

MaFGF + UTMD

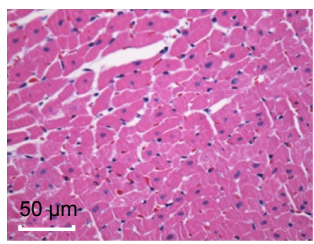

MaFGF-NP + UTMD
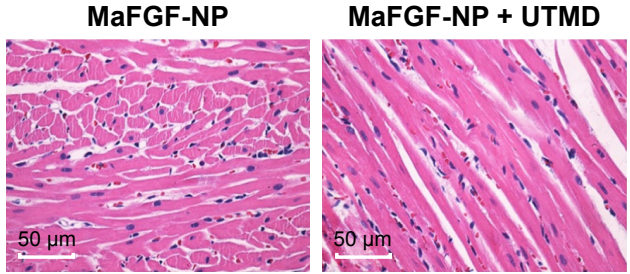

Figure 5 Representative pictures of the hematoxylin and eosin staining (400x).

Abbreviations: DOX-CM, doxorubicin-induced cardiomyopathy; MaFGF-NP, mitogenic acidic fibroblast growth factor-nanoparticles; UTMD, ultrasound-targeted MB destruction.

significantly reduced brown granule deposits $(P<0.05)$ suggesting that the TGF- $\beta 1$ expression was reduced in these groups compared with the DOX-CM group, and the MaFGF-NP + UTMD treated groups showed the lowest expression among all the treatments $(P<0.05)$.

\section{Effects of the MaFGF-NP/UTMD- combined treatment on the electron microscopic findings}

To evaluate the myocardial damage induced by DOX, the ultrastructural changes of the cardiomyocytes were examined. As shown in Figure 8, the distribution of the myocardial damage in the myofibrils, the disorganization of the myofibrillar arrays, the distortion of the mitochondrion and the marked cytoplasmic vacuolization were significantly characterized in the myocardial samples in the DOX-CM animals compared with the controls. In addition, the mitochondria of the cardiomyocytes in the DOX-CM group showed swelling, loss of cristae and disordered arrangement. A similar pattern of damages was observed at the ultrastructural level in the UTMD group. In comparison, few alterations in the myocardial ultrastructure were observed in the MaFGF treatment groups. Furthermore, there were well-organized and symmetric myofibrils and sarcomeres, as well as relatively well-integrated ultrastructures, in the mitochondria in the MaFGF-NP + UTMD group compared with the other treatment and the DOX-CM groups.

\section{Effects of the MaFGF-NP/UTMD- combined treatment on the expression of $\mathrm{Bcl}-2, \mathrm{Bax}$, and $\mathrm{pAkt} / \mathrm{Akt}$ levels}

As shown in Figure 9, the level of proapoptotic protein Bax was up-regulated, while the antiapoptotic protein Bcl-2 was down-regulated in the DOX-CM group compared with the control group $(P<0.05)$. Moreover, the groups treated with MaFGF had a significantly lower level expression of Bax, as well as a higher expression of Bcl-2, compared with the DOX-CM group $(P<0.05)$. Furthermore, there was a significantly up-regulated expression of Bcl-2 and a downregulated expression of Baxin in the rats treated with MaFGF + UTMD compared with the other aFGF treatment groups $(P<0.05)$. The ratio of $\mathrm{pAkt} / \mathrm{Akt}$ in the DOX-CM group was significantly lower than the control groups $(P<0.05)$, while the ratio in the group treated with MaFGF/MaFGF-NP was higher than the DOX-CM groups $(P<0.05)$.

\section{Effects of the MaFGF-NP/UTMD- combined treatment on cardiomyocyte apoptosis}

As shown in Figure 10, the TUNEL staining was performed to estimate the cardiomyocyte apoptosis. The number of apoptotic cells increased significantly after the administration of DOX without any treatment $(P<0.05)$. By contrast, the tissues obtained from the rats in the combined MaFGF-NP and UTMD groups revealed fewer apoptotic cells than the DOX-CM groups $(P<0.05)$. Among the MaFGF treatment groups, there were less apoptotic cells in the MaFGF-NP + UTMD group than the other treatment group. All of the results demonstrated that MaFGF-NP, in combination with UTMD, was an effective antiapoptosis strategy to protect the myocardium from DOX-induced cardiotoxicity.

\section{Discussion}

DOX-CM is the most severe complication of chemotherapy with DOX. ${ }^{2}$ There are a series of available preventive strategies, mostly including supportive treatment or heart 


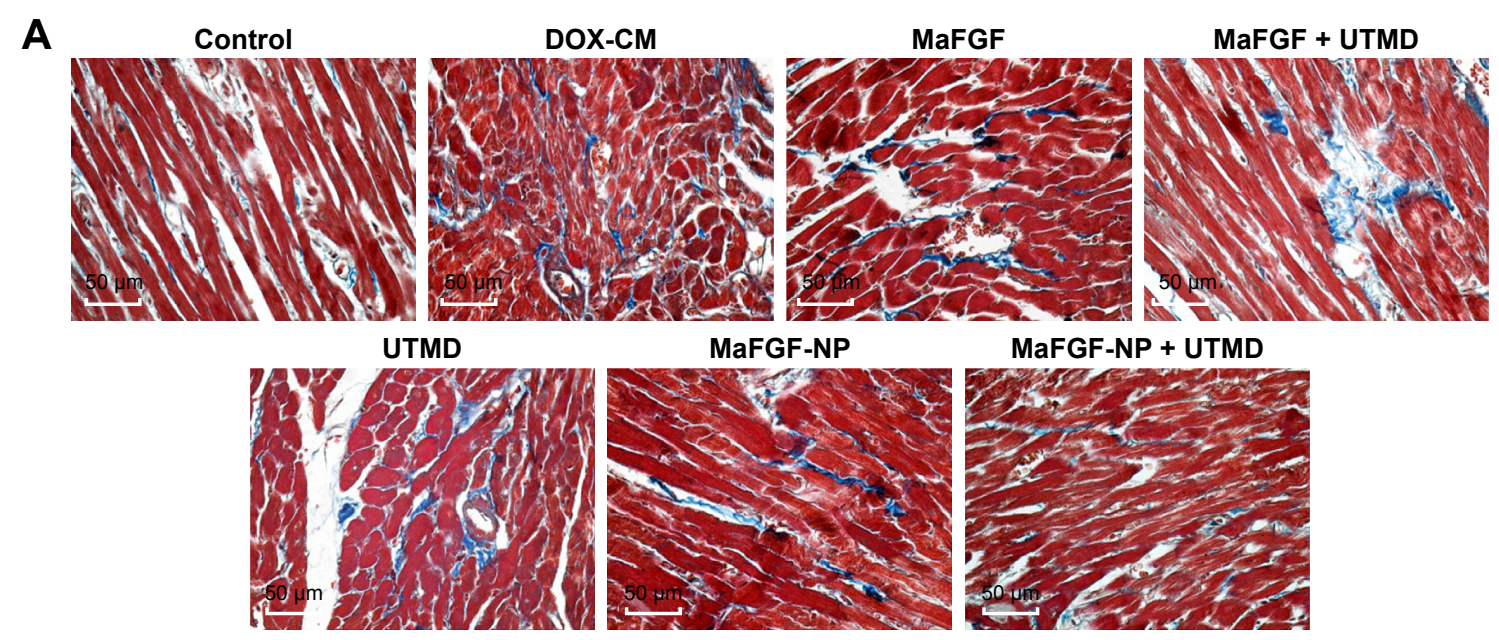

B

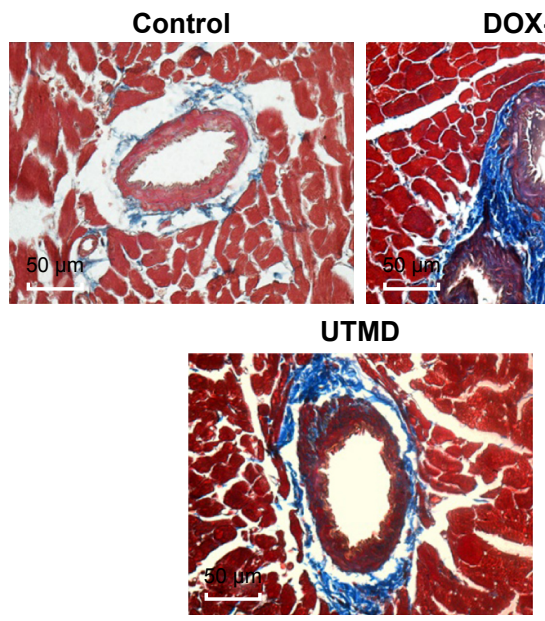

DOX-CM

MaFGF

MaFGF + UTMD

\section{C}

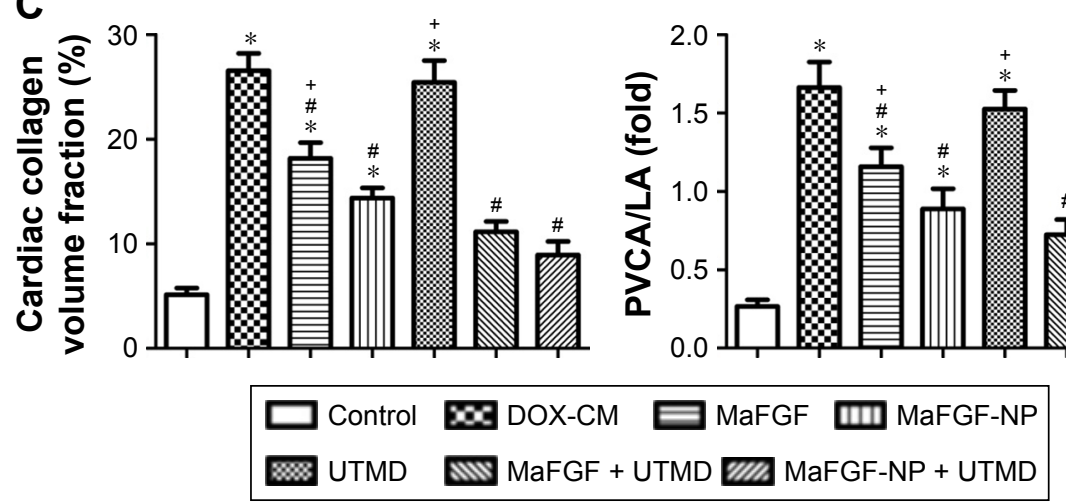

Figure 6 MaFGF-NP combined with UTMD prevented DOX-induced cardiomyocyte interstitial fibrosis and perivascular fibrosis.

Notes: (A, B) Representative pictures of the myocardial tissue sections stained with Masson trichrome showing interstitial and perivascular fibrosis. (C) Quantitative analysis of the cardiac collagen volume fraction (left) and the PVCA/LA (right). ${ }^{* P}<0.05$ vs control group; $\# P<0.05$ vs DOX-CM group; $+P<0.05$ vs MaFGF-NP + UTMD group.

Abbreviations: DOX-CM, doxorubicin-induced cardiomyopathy; MaFGF-NP, mitogenic acidic fibroblast growth factor-nanoparticles; PVCA/LA, perivascular collagen area to luminal area; UTMD, ultrasound-targeted MB destruction.

transplantation, that are adopted to combat DOX-CM, but the efficacies are not satisfactory, and these patients are associated with poor prognosis and worse survival compared to ischemic CM. ${ }^{14}$ Therefore, the development of novel approaches and drugs to effectively prevent this devastating complication of DOX chemotherapy has a great clinical significance.

aFGF has strong mitogenic activities that can induce DNA synthesis in a variety of cell types, promote cell proliferation, growth and differentiation, and promote angiogenesis. ${ }^{15,16}$ 

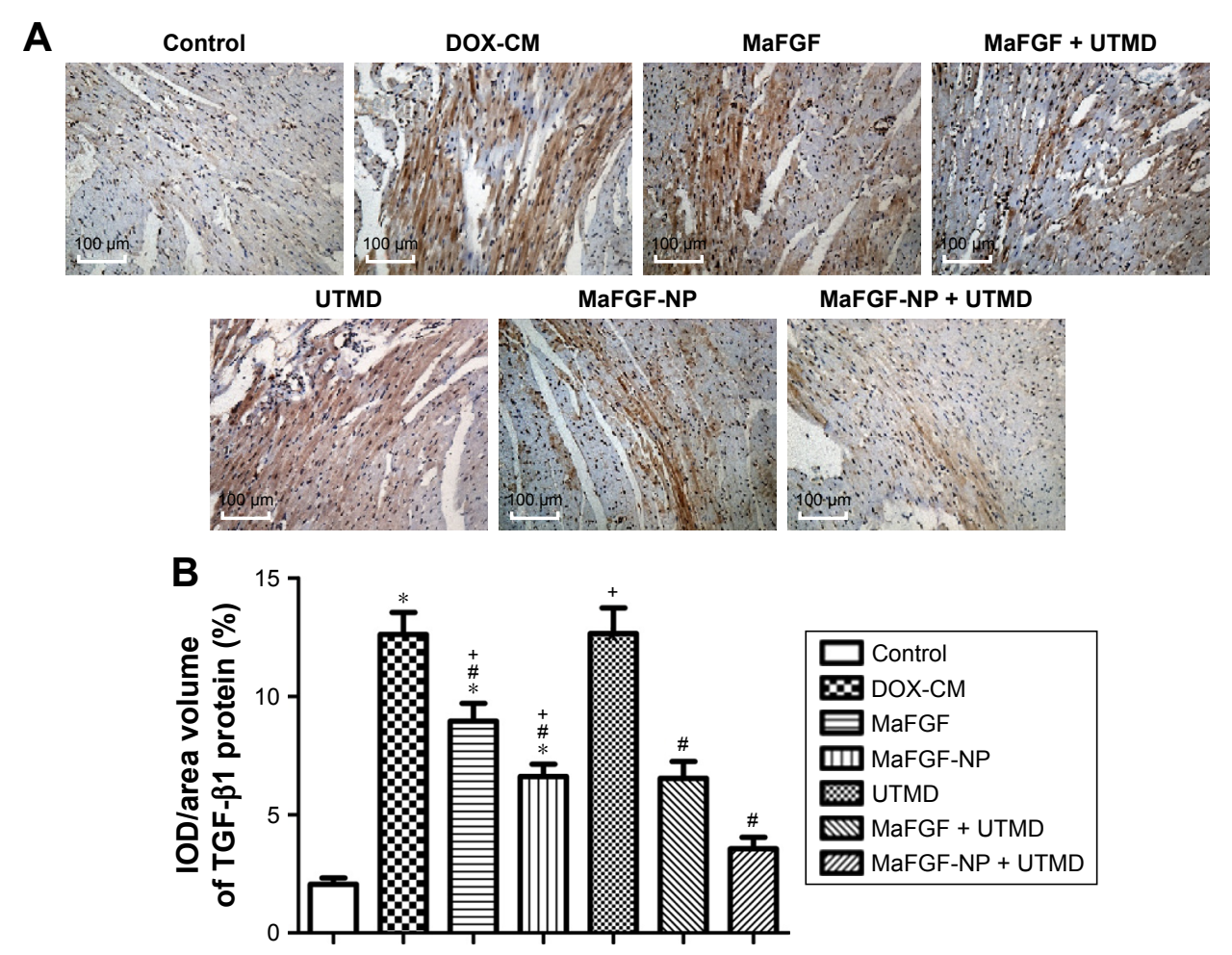

Figure 7 Expression of TGF- $\beta$ I in DOX-induced cardiomyocyte under MaFGF-NP/UTMD combined treatment.

Notes: (A) Representative pictures of immunohistochemical staining of TGF- $\beta$ I expression (200x). (B) Quantitative analysis of the expression of TGF- $\beta$ I. ${ }^{* P}<0.05$ vs control group; ${ }^{P}<0.05$ vs DOX-CM group; ${ }^{+P}<0.05$ vs MaFGF-NP + UTMD group.

Abbreviations: DOX-CM, doxorubicin-induced cardiomyopathy; IOD, integrated optical density; MaFGF-NP, mitogenic acidic fibroblast growth factor-nanoparticles; UTMD, ultrasound-targeted MB destruction.

In addition, aFGF exhibits pleiotropic effects of non-mitogenic activities, such as antioxidation, antiapoptosis, hemangiectasia, neuromodulatory and myocardial protection. All of these effects contribute to its therapeutic effects, such as accelerating wound healing, curing bone damage and protecting the heart, brain, liver, kidney and gut from ischemia and reperfusion injury. ${ }^{8,17}$ However, the powerful mitogenic activities of aFGF are implicated in some pathological processes,
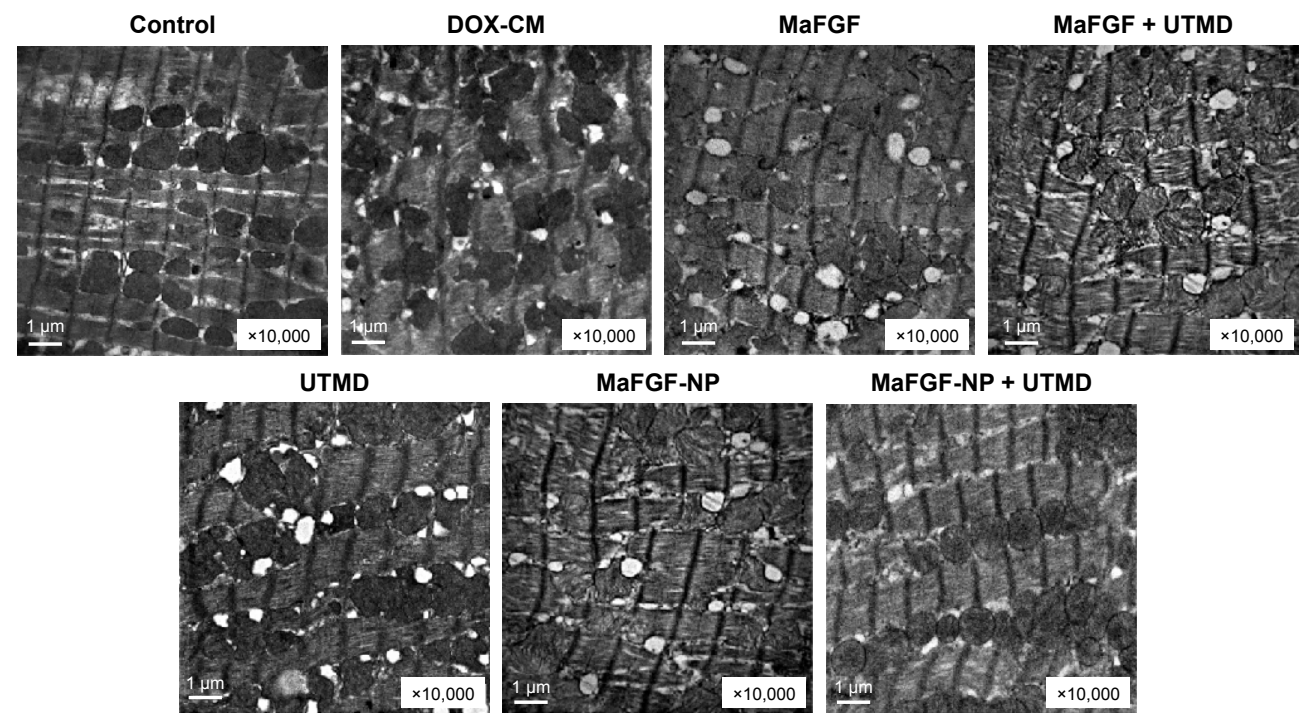

Figure 8 Representative pictures of electron micrographs of left ventricular heart muscle sections from the rats of each group.

Abbreviations: DOX-CM, doxorubicin-induced cardiomyopathy; MaFGF-NP, mitogenic acidic fibroblast growth factor-nanoparticles; UTMD, ultrasound-targeted MB destruction. 

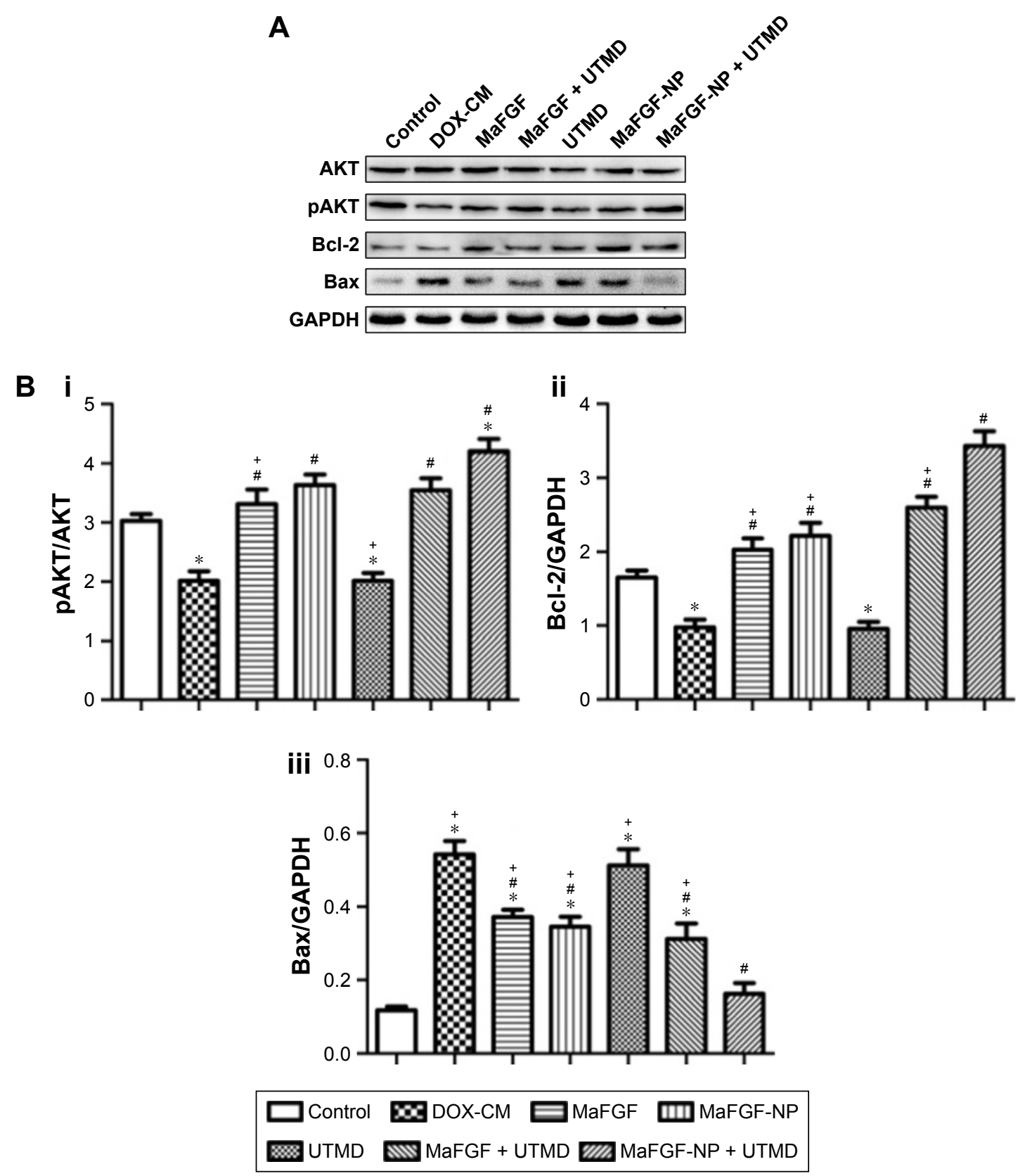

Figure 9 Expression of Akt, pAkt, Bcl-2, Bax and GAPDH in DOX-induced cardiomyocyte under MaFGF-NP/UTMD combined treatment.

Notes: (A) Western blot analysis of the expression of AKT, pAKT, Bcl-2, Bax and GAPDH; (B) Quantitative analyses of the ratio of pAKT/AKT (i), Bcl-2/GAPDH (ii) and Bax/GAPDH (iii). $" P<0.05$ vs DOX-CM group; ${ }^{+P}<0.05$ vs MaFGF-NP + UTMD group.

Abbreviations: DOX-CM, doxorubicin-induced cardiomyopathy; GAPDH, glyceraldehyde 3-phosphate dehydrogenase; MaFGF-NP, mitogenic acidic fibroblast growth factor-nanoparticles; UTMD, ultrasound-targeted MB destruction.

including hyperblastosis, tumorigenesis and metastasis, which became a potential threat to clinical application. ${ }^{6,7}$ MaFGF is a mutant of aFGF that abolishes the mitogenic activity and retains the non-mitogenic activity by a genetically engineered method. ${ }^{18}$ A previous study showed that MaFGF had the same myocardial protective effects as aFGF and can avoid the toxic side effects, such as carcinogenesis. ${ }^{8}$ Nevertheless, MaFGF, as a biological macromolecular drug has a short half-life, poor stability, low bioavailability in systemic circulation and lacks an efficient, safe and controllable myocardial-targeted delivery carrier or method. Meanwhile, the direct injection of MaFGF by an intracoronary, intrapericardial or myocardial injection has many problems, such as an invasive and operational complexity, which limits its clinical application. Previous studies focused on the role of MaFGF in ischemic $\mathrm{CM}$ or diabetic $\mathrm{CM}^{5,8}$ There are few reported studies about MaFGF on non-ischemic CM, such as DOX-CM and heart failure. 
A
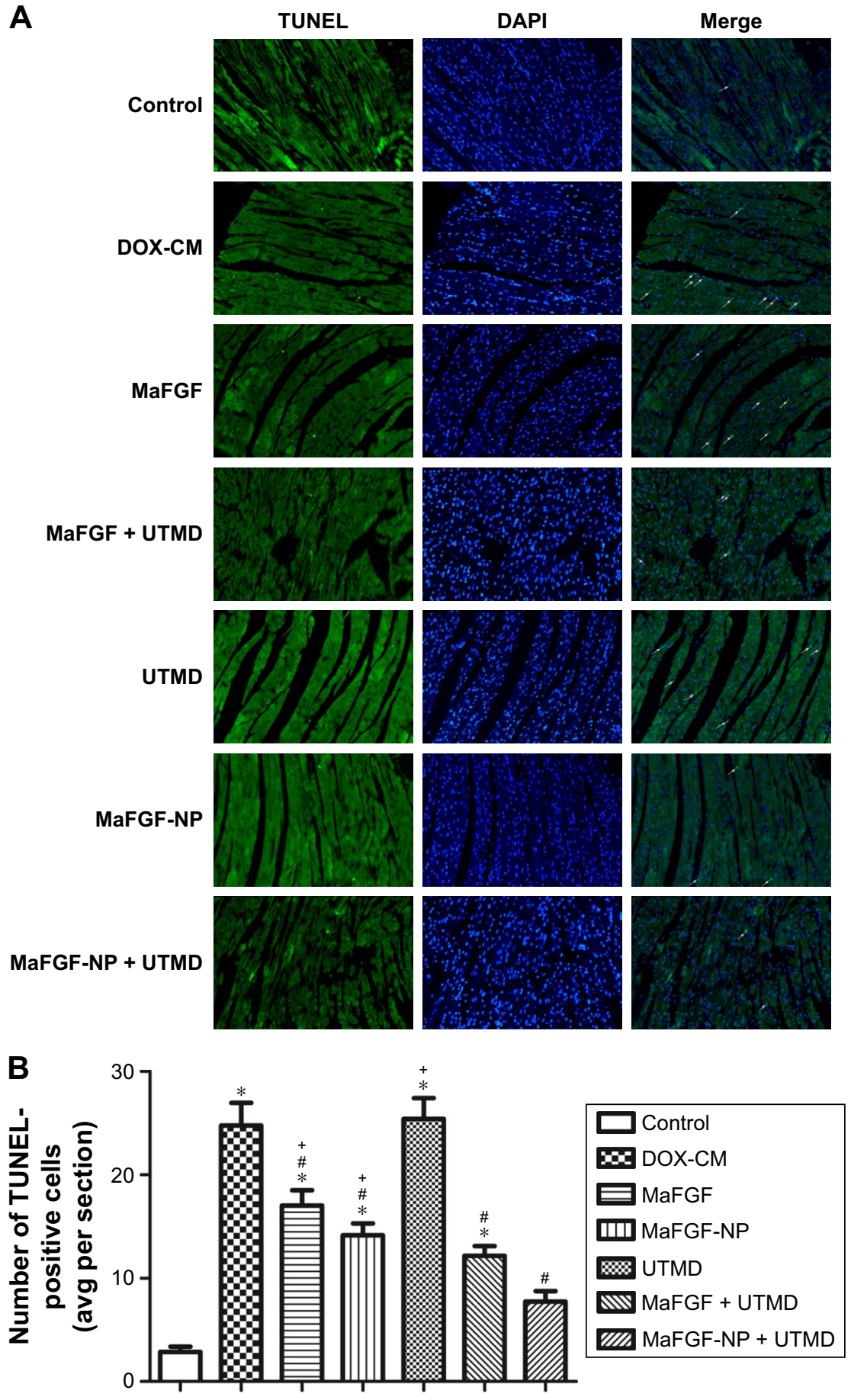

Figure 10 Prevention of DOX-induced cardiomyocytes apoptosis under MaFGF-NP/UTMD combined treatment.

Notes: (A) Representative pictures of the TUNEL staining (200X) (apoptotic cells are indicated by white arrows). (B) Quantitative analysis of the apoptotic cells. $* P<0.05$ vs control group; ${ }^{*} P<0.05$ vs DOX-CM group; ${ }^{+P}<0.05$ vs MaFGF-NP + UTMD group.

Abbreviations: DOX-CM, doxorubicin-induced cardiomyopathy; MaFGF-NP, mitogenic acidic fibroblast growth factor-nanoparticles; TUNEL, terminal deoxynucleotidyl transferase-mediated deoxyuridine triphosphate nick end labelling; UTMD, ultrasound-targeted MB destruction.

UTMD, as a new non-invasive targeted drug and gene delivery technology, has drawn more and more attention. ${ }^{19,20}$ Numerous studies show that UTMD is advantageous because it is non-invasive, has a low immunogenicity and toxicity, has temporal and spatial target specificity and offers repeatability. ${ }^{10,21}$ Ultrasound MBs are used as contrast agents to achieve ultrasound imaging in the regions of interest or targeted areas, and once they are given the proper amplitude and frequency of the ultrasound wave, the cavitation effect called sonoporation causes temporary pore openings in the capillaries and membranes of endothelial cells. Thus, drugs and genes can diffuse through these pores 
and achieve targeted transmission. ${ }^{19,22}$ Chen et al employed UTMD to deliver piggyback transposon plasmids encoding the pancreatic glucagon-like peptide-1 (GLP-1) gene with a nuclear localizing signal to rat hearts with DOX-CM, and the results demonstrated that the UTMD technology successfully delivered the GLP-1 gene targeted to the rat myocardium and reversed the established DOX-CM by stimulating myocardial regeneration. ${ }^{23}$ However, as the carrier of drugs and genes, ultrasound MBs have a low loading capacity, poor stability and high variability. The use of carriers, such as NP, is less risky and can improve the stability of drugs both during storage and in blood circulation. In our previous study, ${ }^{9}$ based on the synergistic effects of NP transportation and MB cavitation, we prepared the aFGF-loaded nanoparticles with high encapsulation efficiency and good bioactivity in combination with UTMD to prevent diabetic cardiomyopathy in a diabetes animal model induced by STZ, and the results showed high aFGF expression in the myocardium and an improved cardiac function.

In the present study, the aim was to evaluate the effects of MaFGF-NP combined with UTMD on DOX-induced cardiac dysfunction and myocardial fibrosis. Consistent with the outcomes of previous studies, ${ }^{24,25}$ the rats with DOX-CM, without MaFGF treatment in the form of decreased BW and increased HW/BW, manifested a severe dysfunction in cardiac performance. Meanwhile, the groups treated with MaFGF-NP + UTMD, during the DOX injury, possessed the lowest HW/BW compared with the untreated DOX-CM animals $(P<0.05)$, which indicates that the MaFGF treatment with NP and UTMD might significantly modify CM.

Echocardiography, as a practical non-invasive tool for measuring cardiac function and structure, is utilized not only in the clinic but also in animals. ${ }^{26}$ In addition, echocardiography is the most commonly used method to detect DOX-CM by measuring the LVEF and LVFS in the clinical setting. In our study, the LV function indices, including LVIDd, LVIDs, EF and FS, were measured by transthoracic echocardiography to estimate global myocardial contractile function. Table 2 shows that the rats administered with DOX and left untreated for 6 weeks were characterized by a declined LVEF and LVFS and an increased LVIDd and LVIDs compared with the control group, and the results confirmed that the cardiotoxicity of DOX significantly caused left ventricle dilation and seriously impaired the diastolic and systolic myocardial performance. In addition, MaFGF-NP + UTMD decreased the LVIDd and LVIDs, as well as increased EF and FS, suggesting that the combination treatment, as an effective strategy, prevented DOX-induced heart function deterioration. VVI is a novel strain analysis technology to assess myocardial function by measuring myocardial deformation using 2D speckle tracking and it is less dependent on volume loading, size, and geometry of the LV. ${ }^{27,28}$ The parameters of myocardial deformation, including the strain and strain rate derived from strain analysis, provide valuable information for detecting early myocardial abnormalities following DOX administration in an animal model. ${ }^{26,29}$ Our study demonstrated that the Vs, RS and RSr were noticeably decreased to various extents in the DOX-CM animals and after treatment with a combination of MaFGF-NP and UTMD, minimal differences, compared with the control, were observed.

The pathophysiology of DOX-CM is not fully elucidated and it is considered to be the result of multiple factors working together that mutually influence increased oxidative stress, inflammation, cardiomyocytes apoptosis, mitochondrial abnormalities, inhibition of nucleic acid, protein synthesis, lysosomal alterations, myocardial electrolytes imbalance, changes in adenylatecyclase, $\mathrm{Na}^{+} / \mathrm{K}^{+}$-adenosine triphosphates (ATPase), and $\mathrm{Ca}^{2+}$ ATPase. ${ }^{3}$ Therefore, increased oxidative stress, through the generation of ROS, is considered to play a major role in DOX-CM. ${ }^{3}$ Cardiac tissues are more vulnerable to oxidative stress caused by DOX-induced cardiotoxicity due to less developed antioxidant defenses, such as SOD1, SOD2, CAT, GSH, and glutathione peroxidase, in the heart compared with other organs, such as the kidney and liver. ${ }^{1}$ SOD is an important antioxidant enzyme that protects the cells from damage by scavenging free radicals, so it plays an important role in the balance of oxidation and antioxidation. The generation of ROS attacks the polyunsaturated fatty acids of biofilm and induces lipid peroxidation followed by lipid peroxides such as MDA. The activity of SOD indirectly reflects the ability of scavenging free radicals, while the level of MDA reflects the severity of the ROS attack. Similar to the outcomes of previously reported studies, ${ }^{30,31}$ we found that the rats in the DOX-CM groups had increased MDA levels and a declined SOD activity, which indicated enhanced oxidative stress in the heart. After a combination treatment with MaFGF-NP and UTMD, the SOD activity significantly improved, and the MDA levels were markedly reduced, suggesting that the alleviated oxidative stress might be due to decreased ROS generation. The mitochondrion is the main organelle of cell energy supplement and respiration, ${ }^{32}$ and the heart has high energy requirement and has a high mitochondrial density, which is more susceptible to DOXinduced lipid peroxidation and toxicity. As shown in the TEM 
images (Figure 8), a distorted condition of the mitochondria was remarkably observed in the DOX-CM groups without any treatment, and the condition was alleviated after the combination treatment, with clear cristae and a wellarranged cardiac fiber ultrastructure, which confirms that MaFGF-NP, combined with UTMD, inhibits the damage caused by oxygen-free radicals to the mitochondria.

A rapidly expanding body of evidence supports the concept that cardiomyocyte apoptosis is involved in DOXinduced toxicity. ${ }^{25,33}$ Moreover, cardiomyocyte apoptosis, and the consequent cardiomyocyte loss, may be a mechanism by which DOX causes deterioration of cardiac function. Akt protects against DOX-induced apoptosis in cardiac myocytes in vitro, and Akt signaling protects against myocyte apoptosis induced by cardiac ischemia-reperfusion injury and diabetic $\mathrm{CM}^{34,35} \mathrm{Xu}$ et al found that MaFGF reduces the level of ActD-induced apoptotic cell death in $20 \mathrm{~h}$, and the protective mechanism of MaFGF may be associated with the activation of the PI3K/Akt signaling pathway by the up-regulation of expression of phosphorylated Akt protein. ${ }^{36}$ The phosphorylated Akt modulates the activities of downstream targets, such as increasing the expression of Bcl-2, which has an anti-apoptotic effect, and decreasing the expression of Bax, which participates in the induction of apoptosis. ${ }^{37}$ In the present study, the Western blot results showed reduction of Akt phosphorylation levels in the heart in the DOX-CM groups, while the level of Bax was up-regulated and that of Bcl-2 was down-regulated compared with the control group. After the treatment with MaFGF/MaFGF-NP, the group treated with MaFGF-NP + UTMD had a significantly higher level expression of phosphorylated Akt and Bcl-2, as well as a lower level expression of Bax, than the DOX-CM group compared with the other treatment groups. Furthermore, we performed TUNEL staining to estimate cardiomyocyte apoptosis, and the results showed a significant increase in TUNEL-positive cells in the DOX-CM group and a decreased number of TUNEL-positive cells in the MaFGF-NP + UTMD group. Therefore, our data indicate that the protective effect of MaFGF-NP, in combination with UTMD, on DOX-CM attenuated cardiomyocyte apoptosis by activating the PI3K/ Akt signaling pathway.

Cardiac fibrosis is the common feature of many cardiac pathophysiological conditions, and this functional alteration results in both systolic and diastolic dysfunction. ${ }^{38}$ Bernaba et al examined the pathology of $\mathrm{CM}$ in patients with anthracycline therapy, and the results showed that the major pathological changes appeared to be interstitial and/or replacement fibrosis without significant cardiac hypertrophy compared with other forms of dilated $\mathrm{CM} .{ }^{39}$ Oxidative stress directly modulates the process of cardiac fibrosis and is also involved in cytokine and growth factor signaling. ${ }^{38}$ Increased oxidative stress activates matrix metalloproteinases and decreases fibrillar collagen synthesis in cardiac fibroblasts. Meanwhile, the activation of TGF- $\beta$, caused by ROS, may enhance extracellular matrix deposition in the cardiac interstitium. ${ }^{40}$ TGF- $\beta$ plays a key role in the development of cardiac fibrosis by inducing the conversion of fibroblasts to myofibroblasts and enhancing extracellular matrix protein synthesis. From the Masson staining and immunohistochemical staining result, the accumulation of collagen in both the interstitial myocardium and perivascular space and the expression of TGF- $\beta 1$ were significantly increased in the DOX-CM rats, and the reductions were observed after MaFGF treatment, especially in the MaFGF-NP + UTMD groups. This is in agreement with the parameter of the transthoracic echocardiography and VVI, and cardiac fibrosis results in increased left ventricular stiffness, decreased compliance, dilated ventricular chamber, followed by left ventricle dysfunction. We concluded that the combination treatment improves left ventricle function and alleviates cardiac fibrosis by disrupting the TGF- $\beta$ signaling pathway.

In conclusion, this study provided extensive in vivo evidence to strongly suggest that the combined application of MaFGF-NP and UTMD effectively prevented myocardial injury induced by DOX and preserved left ventricular systolic function in DOX-CM rats; the mechanism of the combination therapy was involved with antiapoptosis, antioxidant and anti-fibrosis. Therefore, this pre-clinical study provides us with great hope for the future of treating patients with DOX-CM and possibly heart failure.

\section{Acknowledgments}

This research was supported by National Natural Science Foundation of China (Grant No 81571696, 81571392, 81603036 and 81772316), Zhejiang Provincial Natural Science Foundation (Grant No LY17H180008), Zhejiang provincial program for the cultivation of high-level innovative health talents (Ying-Zheng Zhao), 151 talent project of Zhejiang province and 551 talent project of Wenzhou (YingZheng Zhao), key support of high level talent innovation and technology project of Wenzhou (Zhao Ying-Zheng), Zhejiang Provincial Foundation for Health Department (Grant No 2015ZDA023 and 2016KYA136), Medicine Grant from Wenzhou Bureau of Science and Technology (Grant No Y20160086), School Talent Start Fund of Wenzhou Medical University (Grant No QTJ15020). 


\section{Disclosure}

The authors report no conflicts of interest in this work.

\section{References}

1. Menna P, Gonzalez Paz O, Chello M, et al. Anthracycline cardiotoxicity. Expert Opin Drug Saf. 2011;11(Suppl 1):S21-S36.

2. Elliott P. Pathogenesis of cardiotoxicity induced by anthracyclines. Semin Oncol. 2006;33(3 Suppl 8):S2-S7.

3. Carvalho FS, Burgeiro A, Garcia R, Moreno AJ, Carvalho RA, Oliveira PJ. Doxorubicin-induced cardiotoxicity: from bioenergetic failure and cell death to cardiomyopathy. Med Res Rev. 2014;34(1): $106-135$.

4. Xiao J, Lv Y, Lin S, et al. Cardiac protection by basic fibroblast growth factor from ischemia/reperfusion-induced injury in diabetic rats. Biol Pharm Bull. 2010;33(3):444-449.

5. Zhang $\mathrm{C}$, Zhang $\mathrm{L}$, Chen $\mathrm{S}$, et al. The prevention of diabetic cardiomyopathy by non-mitogenic acidic fibroblast growth factor is probably mediated by the suppression of oxidative stress and damage. PLoS One. 2013;8(12):e82287.

6. Jouanneau J, Plouet J, Moens G, Thiery JP. FGF-2 and FGF-1 expressed in rat bladder carcinoma cells have similar angiogenic potential but different tumorigenic properties in vivo. Oncogene. 1997; 14(6):671-676.

7. Facco C, La Rosa S, Dionigi A, Uccella S, Riva C, Capella C. High expression of growth factors and growth factor receptors in ovarian metastases from ileal carcinoids: an immunohistochemical study of 2 cases. Arch Pathol Lab Med. 1998;122(9):828-832.

8. Cuevas P, Carceller F, Lozano RM, Crespo A, Zazo M, GiménezGallego G. Protection of rat myocardium by mitogenic and nonmitogenic fibroblast growth factor during post-ischemic reperfusion. Growth Factors. 1997;15(1):29-40.

9. Zhao YZ, Zhang M, Wong HL, et al. Prevent diabetic cardiomyopathy in diabetic rats by combined therapy of aFGF-loaded nanoparticles and ultrasound-targeted microbubble destruction technique. J Control Release. 2016;223:11-21.

10. Liao YY, Chen ZY, Wang YX, Lin Y, Yang F, Zhou QL. New progress in angiogenesis therapy of cardiovascular disease by ultrasound targeted microbubble destruction. Biomed Res Int. 2014;2014:872984.

11. Zhang Y, Ye C, Wang G, et al. Kidney-targeted transplantation of mesenchymal stem cells by ultrasound-targeted microbubble destruction promotes kidney repair in diabetic nephropathy rats. Biomed Res Int. 2013;2013:526367.

12. Zhu F, Jiang Y, Luo F, Li P. Effectiveness of localized ultrasoundtargeted microbubble destruction with doxorubicin liposomes in H22 mouse hepatocellular carcinoma model. J Drug Target. 2015; 23(4):323-334.

13. Fan CH, Ting CY, Chang YC, Wei KC, Liu HL, Yeh CK. Drug-loaded bubbles with matched focused ultrasound excitation for concurrent blood-brain barrier opening and brain-tumor drug delivery. Acta Biomater. 2015;15:89-101.

14. Felker GM, Thompson RE, Hare JM, et al. Underlying causes and longterm survival in patients with initially unexplained cardiomyopathy. N Engl J Med. 2000;342(15):1077-1084.

15. Meloni M, Descamps B, Caporali A, et al. Nerve growth factor gene therapy using adeno-associated viral vectors prevents cardiomyopathy in type 1 diabetic mice. Diabetes. 2012;61(1):229-240.

16. Engelmann GL, Dionne CA, Jaye MC. Acidic fibroblast growth factor and heart development. Role in myocyte proliferation and capillary angiogenesis. Circ Res. 1993;72(1):7-19.

17. Li HH, Fu XB, Sun TZ, et al. Non-mitogenic acidic fibroblast growth factor reduces intestinal dysfunction induced by ischemia and reperfusion injury in rats. $J$ Gastroenterol Hepatol. 2007;22(3): 363-370.
18. Wu X, Su Z, Li X, Zheng Q, Huang Y, Yuan H. High-level expression and purification of a nonmitogenic form of human acidic fibroblast growth factor in Escherichia coli. Protein Expr Purif. 2005;42(1):7-11.

19. Frenkel V. Ultrasound mediated delivery of drugs and genes to solid tumors. Adv Drug Deliv Rev. 2008;60(10):1193-1208.

20. Zhou ZJ, Ye HY, Cui K, Wang YY. [Ultrasound-mediated microbubble cavitation enhances gene transduction in rat pulmonary endothelial cells partially by affecting membrane fluidity and cytoskeleton structure]. Nan Fang Yi Ke Da Xue Xue Bao. 2010;30(11):2505-2508. Chinese.

21. Sirsi SR, Borden MA. Advances in ultrasound mediated gene therapy using microbubble contrast agents. Theranostics. 2012;2(12): $1208-1222$.

22. Chen ZY, Lin Y, Yang F, Jiang L, Ge Sp. Gene therapy for cardiovascular disease mediated by ultrasound and microbubbles. Cardiovasc Ultrasound. 2013;11:11.

23. Chen S, Chen J, Huang P, et al. Myocardial regeneration in adriamycin cardiomyopathy by nuclear expression of GLP1 using ultrasound targeted microbubble destruction. Biochem Biophys Res Commun. 2015;458(4):823-829.

24. Imbaby S, Ewais M, Essawy S, Faraq N. Cardioprotective effects of curcumin and nebivolol against doxorubicin-induced cardiac toxicity in rats. Hum Exp Toxicol. 2014;33(8):800-813.

25. Ma S, Li X, Dong L, Zhu J, Zhang H, Jia Y. Protective effect of Sheng-Mai Yin, a traditional Chinese preparation, against doxorubicininduced cardiac toxicity in rats. BMC Complement Altern Med. 2016;16:61

26. Migrino RQ, Aggarwal D, Konorev E, Brahmbhatt T, Bright M, Kalyanaraman B. Early detection of doxorubicin cardiomyopathy using two-dimensional strain echocardiography. Ultrasound Med Biol. 2008;34(2):208-214.

27. Zhang H, Wei Z, Zhu X, et al. Assessment of left ventricular myocardial systolic acceleration in diabetic rats using velocity vector imaging. J Ultrasound Med. 2014;33(5):875-883.

28. Chen D, Liao Y, Xu Q, et al. Persistence of systolic and diastolic regional dysfunction after brief episodes of myocardial ischemia evaluated with velocity vector imaging. Int J Cardiol. 2013;167(3): 987-994.

29. Kim YH, Kim M, Park SM, et al. Discordant impairment of multidirectional myocardial deformation in rats with Doxorubicin induced cardiomyopathy. Echocardiography. 2012;29(6):720-728.

30. Kwatra M, Kumar V, Jangra A, et al. Ameliorative effect of naringin against doxorubicin-induced acute cardiac toxicity in rats. Pharm Biol. 2016;54(4):637-647.

31. Mantawy EM, El-Bakly WM, Esmat A, Badr AM, El-Demerdash E. Chrysin alleviates acute doxorubicin cardiotoxicity in rats via suppression of oxidative stress, inflammation and apoptosis. Eur J Pharmacol. 2014;728:107-118.

32. Krause DN, Duckles SP, Pelligrino DA. Influence of sex steroid hormones on cerebrovascular function. J Appl Physiol (1985). 2006; 101(4):1252-1261.

33. Kalyanaraman B, Joseph J, Kalivendi S, Wang S, Konorev E, Kotamraju S. Doxorubicin-induced apoptosis: implications in cardiotoxicity. Mol Cell Biochem. 2002;234-235(1-2):119-124.

34. Chen Y, Ba L, Huang W, et al. Role of carvacrol in cardioprotection against myocardial ischemia/reperfusion injury in rats through activation of MAPK/ERK and Akt/eNOS signaling pathways. Eur $J$ Pharmacol. 2017;796:90-100.

35. Zhang M, Yu WZ, Shen XT, et al. Advanced Interfere Treatment of Diabetic Cardiomyopathy Rats by aFGF-Loaded Heparin-Modified Microbubbles and UTMD Technique. Cardiovasc Drugs Ther. 2016; 30(3):247-261.

36. Xu H, Xu H, Hai GF, He Q, Zhang CE. Protective effect of modified human acidic fibroblast growth factor against actinomycin D-Induced NRK52E Cells Apoptotic death. Tropical J Pharmaceutical Res. $2013 ; 12(3)$. 
37. Chun KH, Lee HY, Hassan K, et al. Implication of protein kinase B/ Akt and Bcl-2/Bcl-XL suppression by the farnesyl transferase inhibitor SCH66336 in apoptosis induction in squamous carcinoma cells. Cancer Res. 2003;63(16):4796-4800.

38. Kong P, Christia P, Frangogiannis NG. The pathogenesis of cardiac fibrosis. Cell Mol Life Sci. 2014;71(4):549-574.
39. Bernaba BN, Chan JB, Lai CK, Fishbein MC. Pathology of lateonset anthracycline cardiomyopathy. Cardiovasc Pathol. 2010;19(5): 308-311.

40. Bujak M, Frangogiannis NG. The role of TGF-beta signaling in myocardial infarction and cardiac remodeling. Cardiovasc Res. 2007; 74(2):184-195.

\section{Publish your work in this journal}

The International Journal of Nanomedicine is an international, peerreviewed journal focusing on the application of nanotechnology in diagnostics, therapeutics, and drug delivery systems throughout the biomedical field. This journal is indexed on PubMed Central, MedLine, CAS, SciSearch $\AA$, Current Contents ${ }^{\circledR} /$ Clinical Medicine,

\section{Dovepress}

Journal Citation Reports/Science Edition, EMBase, Scopus and the Elsevier Bibliographic databases. The manuscript management system is completely online and includes a very quick and fair peer-review system, which is all easy to use. Visit http://www.dovepress.com/ testimonials.php to read real quotes from published authors.

Submit your manuscript here: http://www.dovepress.com/international-journal-of-nanomedicine-journal 